\title{
Entanglement between Two Tavis-Cummings Systems with $\mathbf{N}=\mathbf{2}$
}

\author{
Se ye d Arash Ghore is hi", Mohsen Sarbis haei, Kourosh Javidan \\ Department of Physics, Ferdowsi university of Mashhad, Mashhad, 91775-1436, Iran
}

\begin{abstract}
We consider two non interacting two-atoms Tavis-Cummings systems and examine the entanglement among four two-state atoms. For our purpose, we assume that one atom from each system are entangled initially and we obtain time-dependent concurrence among atoms. There are six pairwise concurrences among atoms and in this paper, we focus on remote atoms. We show that initially non-entangled atoms $A_{2}$ and $B_{2}$ becomes entangled by passing time, the process which may be interpreted as entanglement transfer between the cavities.
\end{abstract}

Keywords Entanglement, Tavis-Cummings Model, Concurrence

\section{Introduction}

Entanglement is one of the most fascinating aspect of quantum mechanics and is a defining future that makes fundamental distinctions between quantum and classical Physics. Two quantum systems A and B are entangled when values of certain properties of system $A$ are correlated with the same properties of system B[1]. Quantum entanglement has been viewed as an essential resources for quantum information process, and a great deal of effort has been devoted to study and characterize the entanglement. Cavity quantum electrodynamics (QED) techniques has been recognized as a promising candidate for the physical realization of quantum information processing[2].

In this paper we consider two non interacting two-ato ms Tavis-Cummings systems and examine the entanglement among four two-states atoms by obtaining the concurrences between each two atoms. The method which we have used is like the method that is used in ref[3]. For this purpose we first introduce the Tavis-Cummings model and then obtain the eigenvalues and eigenstates of the two-atoms Tavis-Cummings system in section 2 . These eigenstates and eigenvalues are used for calculating the time evaluation of entanglement. We introduce the concept of concurrence for measuring the entanglement in section 3 and finally in section 4, we analyze the entanglement among atoms in detail by calculating the reduced density matrice of each two two-levels atoms and give some concludes in section 5 .

* Corresponding author

arashqf@gmail.com (Seyed Arash Ghoreishi)

Published online at http://journal.sapub.org/ijtmp

Copyright (C) 2012 Scientific \& Academic Publishing. All Rights Reserved

\section{The Tavis-Cummings Model}

The Tavis-Cummings model (TCM) describes the simplest fundamental interaction between a single mode of the quantized electromagnetic field and a collection of $\mathrm{N}$ two-level atoms under the rotating wave approximation approximation (RWA) condition[4] -[11]. The Hamiltonian of this model is written as

$$
H_{T C}=\hbar \omega a^{+} a+\frac{1}{2} \hbar \omega \sum_{i=1}^{N} \sigma_{3}^{(i)}+\hbar \lambda \sum_{i=1}^{N}\left(\sigma_{+}^{(i)} a+\sigma_{-}^{(i)} a^{+}\right)
$$

For the case of two-atom $(\mathrm{N}=2)$

$$
\begin{gathered}
H=H_{0}+H_{\mathrm{int}} \\
=\hbar \omega\left(a^{+} a+\frac{1}{2} \sigma_{3}^{(1)}+\frac{1}{2} \sigma_{3}^{(2)}\right) \\
+\hbar \lambda\left[\left(\sigma_{-}^{(1)}+\sigma_{-}^{(2)}\right) a^{+}+\left(\sigma_{+}^{(1)}+\sigma_{+}^{(2)}\right) a\right]
\end{gathered}
$$

Where $a$ and $a^{+}$are the annihilation and creation field operators.

Each in itial state in this system is coupled to three states. For examp le if the initial state is $|i\rangle=\left|e_{1}\right\rangle\left|e_{2}\right\rangle|n\rangle$ then this state is coupled with the states

$$
\begin{aligned}
& \left|f_{1}\right\rangle=\left|e_{1}\right\rangle\left|g_{2}\right\rangle|n+1\rangle \\
& \left|f_{2}\right\rangle=\left|g_{1}\right\rangle\left|e_{2}\right\rangle|n+1\rangle \\
& \left|f_{3}\right\rangle=\left|g_{1}\right\rangle\left|g_{2}\right\rangle|n+2\rangle
\end{aligned}
$$

Writing the Hamiltonian in these states, we obtain 


$$
H^{(n)}=\left(\begin{array}{cccc}
\hbar \omega(n+1) & \hbar \lambda \sqrt{n+2} & \hbar \lambda \sqrt{n+2} & 0 \\
\hbar \lambda \sqrt{n+2} & \hbar \omega(n+1) & 0 & \hbar \lambda \sqrt{n+1} \\
\hbar \lambda \sqrt{n+2} & 0 & \hbar \omega(n+1) & \hbar \lambda \sqrt{n+1} \\
0 & \hbar \lambda \sqrt{n+1} & \hbar \lambda \sqrt{n+1} & \hbar \omega(n+1)
\end{array}\right)
$$

Then the eigenvalues are

$$
\begin{aligned}
& E_{1 n}=\hbar \omega(n+1) \\
& E_{2 n}=\hbar \omega(n+1) \\
& E_{3 n}=\hbar \omega(n+1)+\hbar \lambda \sqrt{2(2 n+3)} \\
& E_{4 n}=\hbar \omega(n+1)-\hbar \lambda \sqrt{2(2 n+3)}
\end{aligned}
$$

and the correlated eigenstates are

$$
\begin{aligned}
& \left|\psi_{1 n}\right\rangle=\sqrt{\frac{n+1}{2 n+3}}\left|g_{1} g_{2} ; n+2\right\rangle-\sqrt{\frac{n+2}{2 n+3}}\left|e_{1} e_{2} ; n\right\rangle \\
& \left|\psi_{2 n}\right\rangle=\frac{1}{\sqrt{2}}\left|g_{1} e_{2} ; n+1\right\rangle-\frac{1}{\sqrt{2}}\left|e_{1} g_{2} ; n+1\right\rangle \\
& \left|\psi_{3 n}\right\rangle=\sqrt{\frac{n+2}{4 n+6}}\left|g_{1} g_{2} ; n+2\right\rangle+\frac{1}{2}\left|g_{1} e_{2} ; n+1\right\rangle \\
& +\frac{1}{2}\left|e_{1} g_{2} ; n+1\right\rangle+\sqrt{\frac{n+1}{4 n+6}}\left|e_{1} e_{2} ; n\right\rangle \\
& \left|\psi_{4 n}\right\rangle=\sqrt{\frac{n+2}{4 n+6}}\left|g_{1} g_{2} ; n+2\right\rangle-\frac{1}{2}\left|g_{1} e_{2} ; n+1\right\rangle \\
& -\frac{1}{2}\left|e_{1} g_{2} ; n+1\right\rangle+\sqrt{\frac{n+1}{4 n+6}}\left|e_{1} e_{2} ; n\right\rangle
\end{aligned}
$$

\section{Measure of Entanglement}

To measure the entanglement, we need to introduce a convenient concept. We will adopt the Wooter's concurrence[13] as our measure:

$$
C(\rho)=\max \left\{0, \sqrt{\lambda_{1}}-\sqrt{\lambda_{2}}-\sqrt{\lambda_{3}}-\sqrt{\lambda_{4}}\right\}
$$

where the quantities $\lambda_{i}$ are the eigenvalues in decreasing order of the matrix

$$
\xi=\rho\left(\sigma_{y} \otimes \sigma_{y}\right) \rho^{*}\left(\sigma_{y} \otimes \sigma_{y}\right)
$$

where $\rho^{*}$ denotes the complex conjugation of $\rho$ in the standard basis and $\sigma_{y}$ is the Pauli matrix.

We may pay attention to this point that although the matrix $\xi$ is not Hermitian, the eigenvalues of this matrix are real and nonnegative.

Note that $1 \geq C \geq 0$, where $C=0$ indicates zero entanglement and $C=1$ means maximal pure state entanglement.

Reduction to a two-qubit form, will yield to a two-qubit mixed state always having the form:

$$
\rho=\left(\begin{array}{cccc}
a & 0 & 0 & z \\
0 & b & 0 & 0 \\
0 & 0 & c & 0 \\
z^{*} & 0 & 0 & d
\end{array}\right)
$$

where $a+b+c+d=1$. The concurrence for this matrix is easily found to be

$$
C=2 \max \{0,|z|-\sqrt{b c}\}
$$

\section{Entanglement Dynamics among Atoms in Two Tavis-Cummings Systems with $\mathbf{N}=\mathbf{2}$}

Consider two two-atoms Tavis-Cummings systems A and $\mathrm{B}$ where $\mathrm{A}$ consists of two atoms $A_{1}, A_{2}$ and $\mathrm{B}$ consists of two atoms $B_{1}, B_{2}$. We assume that initially an atom of system $\mathrm{A}, A_{1}$, is entangled with an atom of system B, $B_{1}$

Now, we are go ing to investigate the entanglement among atoms in this two Tavis-Cummings systems by calculating the concurrence among ato $\mathrm{ms}$. The total init ial state has three parts:

i) The entangled atoms: Th is in itial part can be written in terms of Bell states. We denote this part as follows:

$$
\left|\Phi_{A_{1}, B_{1}}\right\rangle=\cos \alpha\left|e_{1 A} e_{1 B}\right\rangle+\sin \alpha\left|g_{1 A} g_{1 B}\right\rangle
$$

ii) The two atoms which are not entangled initially. We can write this initial part as below:

$$
\begin{aligned}
& \left|\Phi_{A_{2}, B_{2}}(0)\right\rangle=\alpha_{1}\left|e_{2 A} e_{2 B}\right\rangle+\alpha_{2}\left|e_{2 A} g_{2 B}\right\rangle \\
& +\alpha_{3}\left|g_{2 A} e_{2 B}\right\rangle+\alpha_{4}\left|g_{2 A} g_{2 B}\right\rangle
\end{aligned}
$$

To make sure that these atoms are not entangled initially, we must have

$$
\alpha_{1} \alpha_{4}=\alpha_{2} \alpha_{3}
$$

iii) The cavities Initial states:

$$
\left|\Phi_{F}(0)\right\rangle=|n\rangle_{A} \otimes|m\rangle_{B}
$$

where $\mathrm{n}(\mathrm{m})$ is the number of photons in cavities A $(\mathrm{B})$ at $\mathrm{t}=0$.

Thus the total initial state is

$$
\begin{aligned}
|\Phi(0)\rangle & =\left(\cos \alpha\left|e_{1 A} e_{1 B}\right\rangle+\sin \alpha\left|g_{1 A} g_{1 B}\right\rangle\right) \\
\otimes & \left(\alpha_{1}\left|e_{2 A} e_{12 B}\right\rangle+\alpha_{2}\left|e_{2 A} g_{2 B}\right\rangle\right) \\
+ & \alpha_{3}\left|g_{2 A} e_{2 B}\right\rangle+\alpha_{4}\left|g_{2 A} g_{2 B}\right\rangle
\end{aligned}
$$

which can be written as

$$
\begin{aligned}
|\Phi(0)\rangle & =\cos \alpha\left[\alpha_{1}\left|e_{1} e_{2} ; n\right\rangle_{A} \otimes\left|e_{1} e_{2} ; m\right\rangle_{B}\right. \\
& +\alpha_{2}\left|e_{1} e_{2} ; n\right\rangle_{A} \otimes\left|e_{1} g_{2} ; m\right\rangle_{B} \\
& +\alpha_{3}\left|e_{1} g_{2} ; n\right\rangle_{A} \otimes\left|e_{1} e_{2} ; m\right\rangle_{B} \\
& \left.+\alpha_{4}\left|e_{1} g_{2} ; n\right\rangle_{A} \otimes\left|e_{1} g_{2} ; m\right\rangle_{B}\right]
\end{aligned}
$$




$$
\begin{gathered}
+\sin \alpha\left[\alpha_{1}\left|g_{1} e_{2} ; n\right\rangle_{A} \otimes\left|g_{1} e_{2} ; m\right\rangle_{B}\right. \\
+\alpha_{2}\left|g_{1} e_{2} ; n\right\rangle_{A} \otimes\left|g_{1} g_{2} ; m\right\rangle_{B} \\
+\alpha_{3}\left|g_{1} g_{2} ; n\right\rangle_{A} \otimes\left|g_{1} e_{2} ; m\right\rangle_{B} \\
\left.+\alpha_{4}\left|g_{1} g_{2} ; n\right\rangle_{A} \otimes\left|g_{1} g_{2} ; m\right\rangle_{B}\right]
\end{gathered}
$$

To simplify our calculations, we suppose that the non entangled atoms are initially in excited states $\left(\alpha_{1}=1\right.$, $\left.\alpha_{2}=\alpha_{3}=\alpha_{4}=0\right)$ and the number of photons in two cavities are equal $(\mathrm{n}=\mathrm{m})$. By applying these conditions, the initial state becomes

$$
\begin{aligned}
|\Phi(0)\rangle & =\cos \alpha\left|e_{1} e_{2} ; n\right\rangle_{A} \otimes\left|e_{1} e_{2} ; n\right\rangle_{B} \\
& +\sin \alpha\left|g_{1} e_{2} ; n\right\rangle_{A} \otimes\left|g_{1} e_{2} ; n\right\rangle_{B}
\end{aligned}
$$

To prepare for the time evolution we express these states in terms of the eigenstates given in (6), and obtain

$$
\begin{aligned}
& \left|e_{1} e_{2} ; n\right\rangle=a_{1 n}\left|\psi_{1 n}\right\rangle+a_{3 n}\left|\psi_{3 n}\right\rangle+a_{4 n}\left|\psi_{4 n}\right\rangle \\
& \left|g_{1} g_{2} ; n\right\rangle=b_{1 n}\left|\psi_{1(n-2)}\right\rangle+b_{3 n}\left|\psi_{3(n-2)}\right\rangle+b_{4 n}\left|\psi_{4(n-2)}\right\rangle \\
& \left|g_{1} e_{2} ; n\right\rangle=c_{2 n}\left|\psi_{2(n-1)}\right\rangle+c_{3 n}\left|\psi_{3(n-1)}\right\rangle+c_{4 n}\left|\psi_{4(n-1)}\right\rangle \\
& \left|e_{1} g_{2} ; n\right\rangle=d_{2 n}\left|\psi_{2(n-1)}\right\rangle+d_{3 n}\left|\psi_{3(n-1)}\right\rangle+d_{4 n}\left|\psi_{4(n-1)}\right\rangle
\end{aligned}
$$

where

$$
\begin{gathered}
a_{1 n}=-\sqrt{\frac{n+2}{2 n+3}} a_{3 n}=a_{4 n}=\sqrt{\frac{n+1}{4 n+6}} \\
b_{1 n}=\sqrt{\frac{n-1}{2 n-1}} b_{3 n}=b_{4 n}=\sqrt{\frac{n}{4 n-2}} \\
c_{2 n}=\frac{1}{\sqrt{2}} c_{3 n}=\frac{1}{2} c_{4 n}=-\frac{1}{2} \\
d_{2 n}=-\frac{1}{\sqrt{2}} d_{3 n}=\frac{1}{2} \quad d_{4 n}=-\frac{1}{2}
\end{gathered}
$$

Thus, the initial state is

$$
\begin{gathered}
|\Phi(0)\rangle=\cos \alpha\left(a_{1 n}\left|\psi_{1 n}\right\rangle_{A}+a_{3 n}\left|\psi_{3 n}\right\rangle_{A}+a_{4 n}\left|\psi_{4 n}\right\rangle_{A}\right) \\
\otimes\left(a_{1 n}\left|\psi_{1 n}\right\rangle_{B}+a_{3 n}\left|\psi_{3 n}\right\rangle_{B}+a_{4 n}\left|\psi_{4 n}\right\rangle_{B}\right) \\
+\sin \alpha\left(c_{2 n}\left|\psi_{2(n-1)}\right\rangle_{A}+c_{3 n}\left|\psi_{3(n-1)}\right\rangle_{A}+c_{4 n}\left|\psi_{4(n-1)}\right\rangle_{\mathrm{A}}\right) \\
\otimes\left(c_{2 n}\left|\psi_{2(n-1)}\right\rangle_{B}+c_{3 n}\left|\psi_{3(n-1)}\right\rangle_{B}+c_{4 n}\left|\psi_{4(n-1)}\right\rangle_{\mathrm{B}}\right)
\end{gathered}
$$

Since the time evolution of these eigenstates is specified we can transfer these time evolutions to the coefficients. Therefore

$$
\begin{aligned}
& |\Phi(t)\rangle=\cos \alpha\left(a_{1 n}(t)\left|\psi_{1 n}\right\rangle_{A}+a_{3 n}(t)\left|\psi_{3 n}\right\rangle_{A}\right. \\
& \left.+a_{4 n}(t)\left|\psi_{4 n}\right\rangle_{A}\right) \\
& \otimes\left(a_{1 n}(t)\left|\psi_{1 n}\right\rangle_{B}+a_{3 n}(t)\left|\psi_{3 n}\right\rangle_{B}+a_{4 n}(t)\left|\psi_{4 n}\right\rangle_{B}\right) \\
& +\sin \alpha\left(c_{2 n}(t)\left|\psi_{2(n-1)}\right\rangle_{A}+c_{3 n}(t)\left|\psi_{3(n-1)}\right\rangle_{A \mathrm{~A}}\right.
\end{aligned}
$$

$$
\begin{gathered}
+c_{4 n}(t)\left|\psi_{4(n-1)}\right\rangle \\
\otimes\left(c_{2 n}(t)\left|\psi_{2(n-1)}\right\rangle_{B}+c_{3 n}(t)\left|\psi_{3(n-1)}\right\rangle_{B}+c_{4 n}(t)\left|\psi_{4(n-1)}\right\rangle_{\mathrm{B}}\right)
\end{gathered}
$$

In order to take traces over individual ato ms or cavities, we need to revert to the bare bases and this leads to

$$
\begin{aligned}
& |\Phi(t)\rangle=\left[\left(\beta_{1 n} a_{1 n}(t)+\frac{1}{\sqrt{2}} \beta_{2 n}\left(a_{3 n}(t)+a_{4 n}(t)\right)\right)\right. \\
& \times \cos \alpha\left|g_{1} g_{2} ; n+2\right\rangle_{A} \\
& +\left(-\beta_{2 n} a_{1 n}(t)+\frac{1}{\sqrt{2}} \beta_{1 n}\left(a_{3 n}(t)+a_{4 n}(t)\right)\right) \\
& \times \cos \alpha\left|e_{1} e_{2} ; n\right\rangle_{A} \\
& +\frac{1}{2}\left(a_{3 n}(t)-a_{4 n}(t)\right) \cos \alpha\left|g_{1} e_{2} ; n+1\right\rangle_{A} \\
& +\frac{1}{2}\left(a_{3 n}(t)-a_{4 n}(t)\right) \cos \alpha\left|g_{1} e_{2} ; n+1\right\rangle_{A} \\
& \otimes\left[\left(\beta_{1 n} a_{1 n}(t)+\frac{1}{\sqrt{2}} \beta_{2 n}\left(a_{3 n}(t)+a_{4 n}(t)\right)\right)\right. \\
& \times \cos \alpha\left|g_{1} g_{2} ; n+2\right\rangle_{B} \\
& +\left(-\beta_{2 n} a_{1 n}(t)+\frac{1}{\sqrt{2}} \beta_{1 n}\left(a_{3 n}(t)+a_{4 n}(t)\right)\right) \\
& \times \cos \alpha\left|e_{1} e_{2} ; n\right\rangle_{B} \\
& +\frac{1}{2}\left(a_{3 n}(t)-a_{4 n}(t)\right) \cos \alpha\left|g_{1} e_{2} ; n+1\right\rangle_{B} \\
& \left.+\frac{1}{2}\left(a_{3 n}(t)-a_{4 n}(t)\right) \cos \alpha\left|g_{1} e_{2} ; n+1\right\rangle_{B}\right] \\
& +\left[\left(\frac{1}{\sqrt{2}} c_{2 n}(t)+\frac{1}{2} c_{3 n}(t)-\frac{1}{2} c_{4 n}(t)\right) \sin \alpha\left|g_{1} e_{2} ; n\right\rangle_{A}\right. \\
& +\left(-\frac{1}{\sqrt{2}} c_{2 n}(t)+\frac{1}{2} c_{3 n}(t)-\frac{1}{2} c_{4 n}(t)\right) \sin \alpha\left|g_{1} e_{2} ; n\right\rangle_{A} \\
& +\frac{\gamma_{2 n}}{\sqrt{2}}\left(c_{3 n}(t)+c_{4 n}(t)\right) \sin \alpha\left|g_{1} g_{2} ; n+1\right\rangle_{A} \\
& \left.+\frac{\gamma_{1 n}}{\sqrt{2}}\left(c_{3 n}(t)+c_{4 n}(t)\right) \sin \alpha\left|e_{1} e_{2} ; n-1\right\rangle_{A}\right] \\
& \otimes\left[\left(\frac{1}{\sqrt{2}} c_{2 n}(t)+\frac{1}{2} c_{3 n}(t)-\frac{1}{2} c_{4 n}(t)\right) \sin \alpha\left|g_{1} e_{2} ; n\right\rangle_{B}\right. \\
& +\left(-\frac{1}{\sqrt{2}} c_{2 n}(t)+\frac{1}{2} c_{3 n}(t)-\frac{1}{2} c_{4 n}(t)\right) \sin \alpha\left|g_{1} e_{2} ; n\right\rangle_{B} \\
& +\frac{\gamma_{2 n}}{\sqrt{2}}\left(c_{3 n}(t)+c_{4 n}(t)\right) \sin \alpha\left|g_{1} g_{2} ; n+1\right\rangle_{B} \\
& \left.+\frac{\gamma_{1 n}}{\sqrt{2}}\left(c_{3 n}(t)+c_{4 n}(t)\right) \sin \alpha\left|e_{1} e_{2} ; n-1\right\rangle_{B}\right]
\end{aligned}
$$


where

$$
\begin{gathered}
\beta_{1 n}=\sqrt{\frac{n+1}{2 n+3}} \\
\beta_{2 n}=\sqrt{\frac{n+2}{2 n+3}} \\
\gamma_{1 n}=\sqrt{\frac{n}{2 n+1}} \\
\gamma_{2 n}=\sqrt{\frac{n+1}{2 n+1}}
\end{gathered}
$$

For simplicity, we define $\rho^{\text {atoms }}$ by trace out from density operator on the photonic states

$$
\begin{gathered}
\rho^{\text {atoms }}=\langle n+2 ; n+2 \mid \Phi(t)\rangle\langle\Phi(t) \mid n+2 ; n+2\rangle \\
+\langle n+2 ; n+1 \mid \Phi(t)\rangle\langle\Phi(t) \mid n+2 ; n+1\rangle \\
+\langle n+2 ; n \mid \Phi(t)\rangle\langle\Phi(t) \mid n+2 ; n\rangle \\
+\langle n+2 ; n-1 \mid \Phi(t)\rangle\langle\Phi(t) \mid n+2 ; n-1\rangle \\
+\langle n+1 ; n+2 \mid \Phi(t)\rangle\langle\Phi(t) \mid n+1 ; n+2\rangle \\
+\langle n+1 ; n+1 \mid \Phi(t)\rangle\langle\Phi(t) \mid n+1 ; n+1\rangle \\
+\langle n+1 ; n \mid \Phi(t)\rangle\langle\Phi(t) \mid n+1 ; n\rangle \\
+\langle n+1 ; n-1 \mid \Phi(t)\rangle\langle\Phi(t) \mid n+1 ; n-1\rangle \\
+\langle n ; n+2 \mid \Phi(t)\rangle\langle\Phi(t) \mid n ; n+2\rangle \\
+\langle n ; n+1 \mid \Phi(t)\rangle\langle\Phi(t) \mid n ; n+1\rangle \\
+\langle n ; n \mid \Phi(t)\rangle\langle\Phi(t) \mid n ; n\rangle \\
+\langle n ; n-1 \mid \Phi(t)\rangle\langle\Phi(t) \mid n ; n-1\rangle \\
+\langle n-1 ; n+2 \mid \Phi(t)\rangle\langle\Phi(t) \mid n-1 ; n+2\rangle \\
+\langle n-1 ; n+1 \mid \Phi(t)\rangle\langle\Phi(t) \mid n-1 ; n+1\rangle \\
+\langle n-1 ; n \mid \Phi(t)\rangle\langle\Phi(t) \mid n-1 ; n\rangle \\
+\langle n-1 ; n-1 \mid \Phi(t)\rangle\langle\Phi(t) \mid n-1 ; n-1\rangle
\end{gathered}
$$

Now, by using $\rho^{\text {atoms }}$ we can obtain the reduced density matrix between each two ato ms.

We first focus on $C^{A_{1} B_{1}}$. This requires that we trace out $A_{2}$ and $B_{2}$ atom states, in order to get the two-qubit mixed state needed for calculation at $A_{1} B_{1}$ concurrence. The $\rho^{A_{1} B_{1}}$ becomes

$$
\begin{gathered}
\rho^{A_{1} B_{1}}=\left\langle e_{2 A}, e_{2 B}\left|\rho^{\text {atoms }}\right| e_{2 A}, e_{2 B}\right\rangle \\
+\left\langle e_{2 A}, g_{2 B}\left|\rho^{\text {atoms }}\right| e_{2 A}, g_{2 B}\right\rangle \\
+\left\langle g_{2 A}, e_{2 B}\left|\rho^{\text {atoms }}\right| g_{2 A}, e_{2 B}\right\rangle \\
+\left\langle g_{2 A}, g_{2 B}\left|\rho^{\text {atoms }}\right| g_{2 A}, g_{2 B}\right\rangle
\end{gathered}
$$

We see that the $A_{1} B_{1}$ mixed state has the form

$$
\rho^{A_{1} B_{1}}=\left(\begin{array}{cccc}
a & 0 & 0 & z \\
0 & b & 0 & 0 \\
0 & 0 & c & 0 \\
z^{*} & 0 & 0 & d
\end{array}\right)
$$

for which the concurrence has the stated form

$$
C^{A_{1} B_{1}}=2 \max \{0,|z|-\sqrt{b c}\}
$$

with

$$
\begin{gathered}
z=\left(2 x_{6} x_{7}^{*}+x_{12} x_{13}^{*}+x_{4} x_{5}^{*}\right) \sin \alpha \cos \alpha \\
b=c=\left(\left|x_{2}\right|^{2}+\left|x_{3}\right|^{2}+\left|x_{4}\right|^{2}+\left|x_{6}\right|^{2}\right) \cos ^{2} \alpha \\
+\left(\left|x_{8}\right|^{2}+\left|x_{9}\right|^{2}+\left|x_{12}\right|^{2}+\left|x_{14}\right|^{2}\right) \sin ^{2} \alpha
\end{gathered}
$$

The time dependent coefficients are

$$
\begin{aligned}
& x_{1}=\left(\beta_{1} a_{1}(t)+\frac{1}{\sqrt{2}} \beta_{2}\left(a_{3}(t)+a_{4}(t)\right)\right)^{2} \\
& x_{2}=\frac{1}{2}\left(\beta_{1} a_{1}(t)+\frac{1}{\sqrt{2}} \beta_{2}\left(a_{3}(t)+a_{4}(t)\right)\right)\left(a_{3}(t)-a_{4}(t)\right) \\
& x_{3}=\frac{1}{2}\left(\beta_{1} a_{1}(t)+\frac{1}{\sqrt{2}} \beta_{2}\left(a_{3}(t)+a_{4}(t)\right)\right) \\
& \times\left(-\beta_{2} a_{1}(t)+\frac{1}{\sqrt{2}} \beta_{1}\left(a_{3}(t)+a_{4}(t)\right)\right) \\
& x_{4}=\frac{1}{4}\left(a_{3}(t)-a_{4}(t)\right)^{2} \\
& x_{5}=\frac{\gamma_{2}^{2}}{2}\left(c_{3}(t)+c_{4}(t)\right)^{2} \\
& x_{6}=\frac{1}{2}\left(a_{3}(t)-a_{4}(t)\right) \\
& \times\left(-\beta_{2} a_{1}(t)+\frac{1}{\sqrt{2}} \beta_{1}\left(a_{3}(t)+a_{4}(t)\right)\right) \\
& x_{7}=\frac{\gamma_{2}}{\sqrt{2}}\left(c_{3}(t)+c_{4}(t)\right)\left(\frac{1}{\sqrt{2}} c_{2}(t)+\frac{1}{2} c_{3}(t)-\frac{1}{2} c_{4}(t)\right) \\
& x_{8}=\frac{\gamma_{2}}{\sqrt{2}}\left(c_{3}(t)+c_{4}(t)\right)\left(-\frac{1}{\sqrt{2}} c_{2}(t)+\frac{1}{2} c_{3}(t)-\frac{1}{2} c_{4}(t)\right) \\
& x_{9}=\frac{1}{2} \gamma_{1} \gamma_{2}\left(c_{3}(t)+c_{4}(t)\right)^{2} \\
& x_{10}=\left(-\beta_{2} a_{1}(t)+\frac{1}{\sqrt{2}} \beta_{1}\left(a_{3}(t)+a_{4}(t)\right)\right)^{2} \\
& x_{11}=\left(\frac{1}{\sqrt{2}} c_{2}(t)+\frac{1}{2} c_{3}(t)-\frac{1}{2} c_{4}(t)\right)^{2}
\end{aligned}
$$




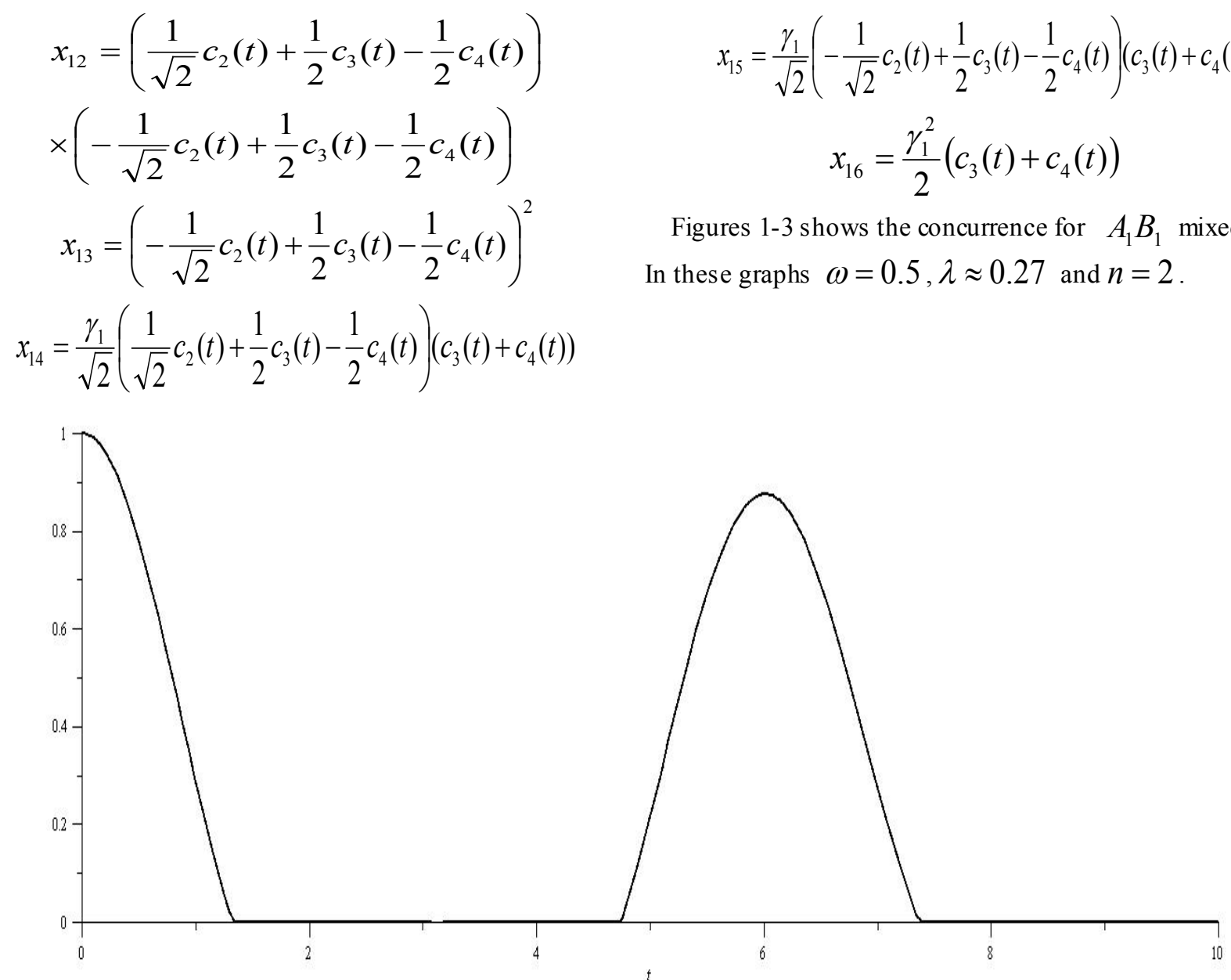

Figure 1. plot of $A_{1} B_{1}$ concurrence for $\alpha=\frac{\pi}{4}$

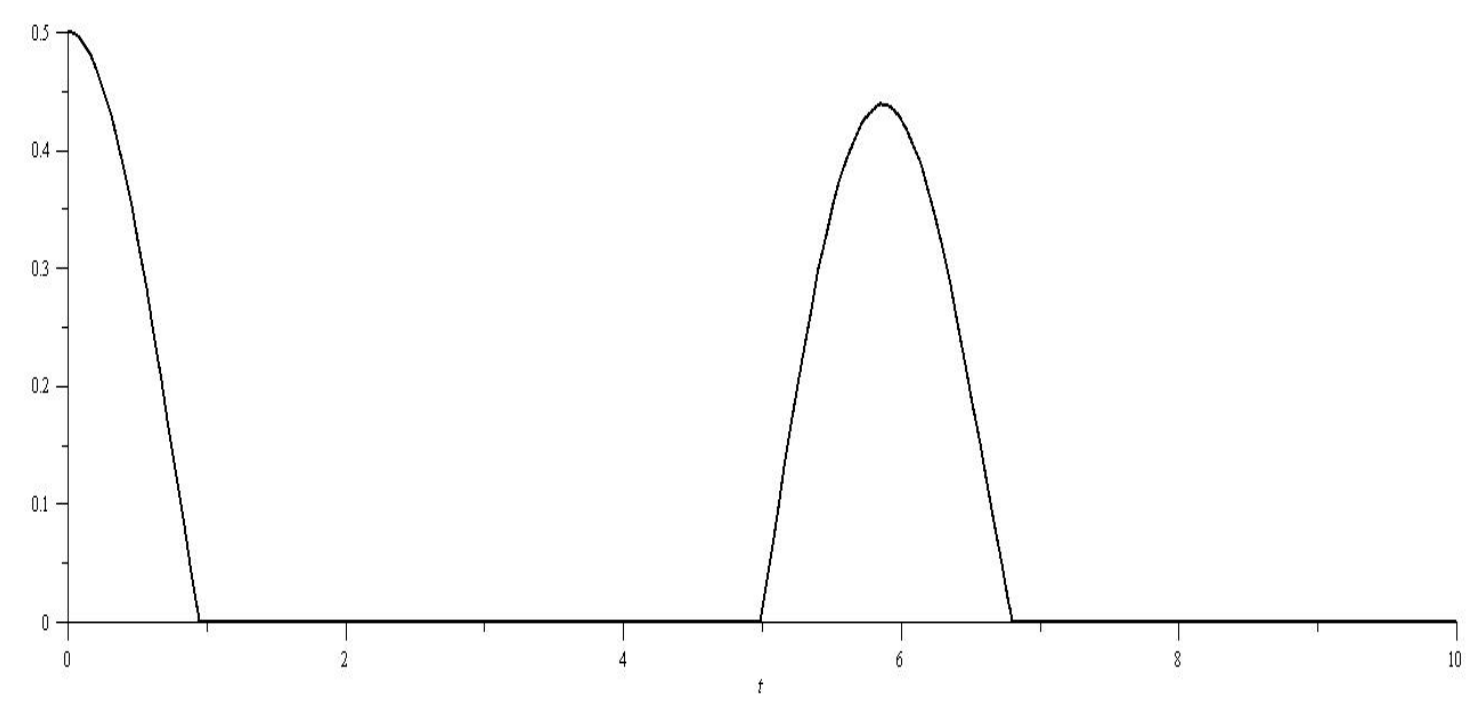

Figure 2. plot of $A_{1} B_{1}$ concurrence for $\alpha=\frac{\pi}{12}$ 


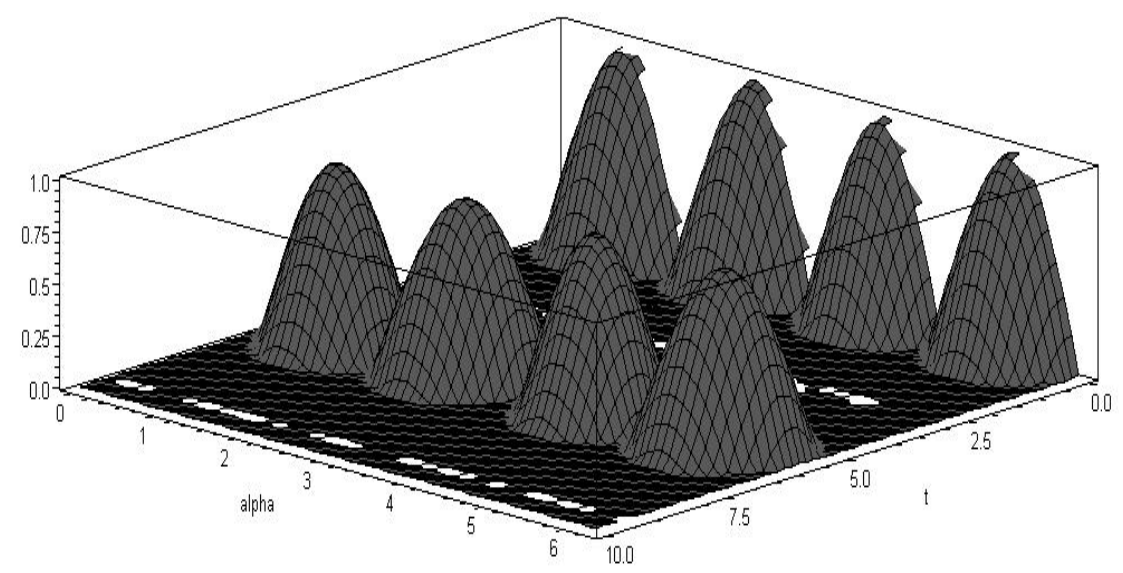

Figure 3. Surface plot of the concurrence

For concurrence calculation between $A_{2}$ and $B_{2}$ :

$$
\begin{gathered}
\rho^{A_{2} B_{2}}=\left\langle e_{1 A}, e_{1 B}\left|\rho^{\text {atoms }}\right| e_{1 A}, e_{1 B}\right\rangle \\
+\left\langle e_{1 A}, g_{1 B}\left|\rho^{\text {atoms }}\right| e_{1 A}, g_{1 B}\right\rangle \\
+\left\langle g_{1 A}, e_{1 B}\left|\rho^{\text {atoms }}\right| g_{1 A}, e_{1 B}\right\rangle \\
+\left\langle g_{1 A}, g_{1 B}\left|\rho^{\text {atoms }}\right| g_{1 A}, g_{1 B}\right\rangle
\end{gathered}
$$

and we find

$$
\begin{aligned}
z & =\left(x_{4} x_{5}^{*}+x_{10} x_{13}^{*}+2 x_{6} x_{8}^{*}\right) \sin \alpha \cos \alpha \\
b & =\left(\left|x_{2}\right|^{2}+\left|x_{3}\right|^{2}+\left|x_{4}\right|^{2}+\left|x_{8}\right|^{2}\right) \cos ^{2} \alpha \\
+ & \left(\left|x_{8}\right|^{2}+\left|x_{9}\right|^{2}+\left|x_{12}\right|^{2}+\left|x_{14}\right|^{2}\right) \sin ^{2} \alpha \\
c & =\left(\left|x_{2}\right|^{2}+\left|x_{3}\right|^{2}+\left|x_{4}\right|^{2}+\left|x_{6}\right|^{2}\right) \cos ^{2} \alpha \\
& +\left(\left|x_{7}\right|^{2}+\left|x_{9}\right|^{2}+\left|x_{14}\right|^{2}+\left|x_{15}\right|^{2}\right) \sin ^{2} \alpha
\end{aligned}
$$

Figures 4 and 5 show the plots for this concurrence for $\alpha=\frac{\pi}{4}$ and $\alpha=\frac{\pi}{12}$ at the first 100 seconds.

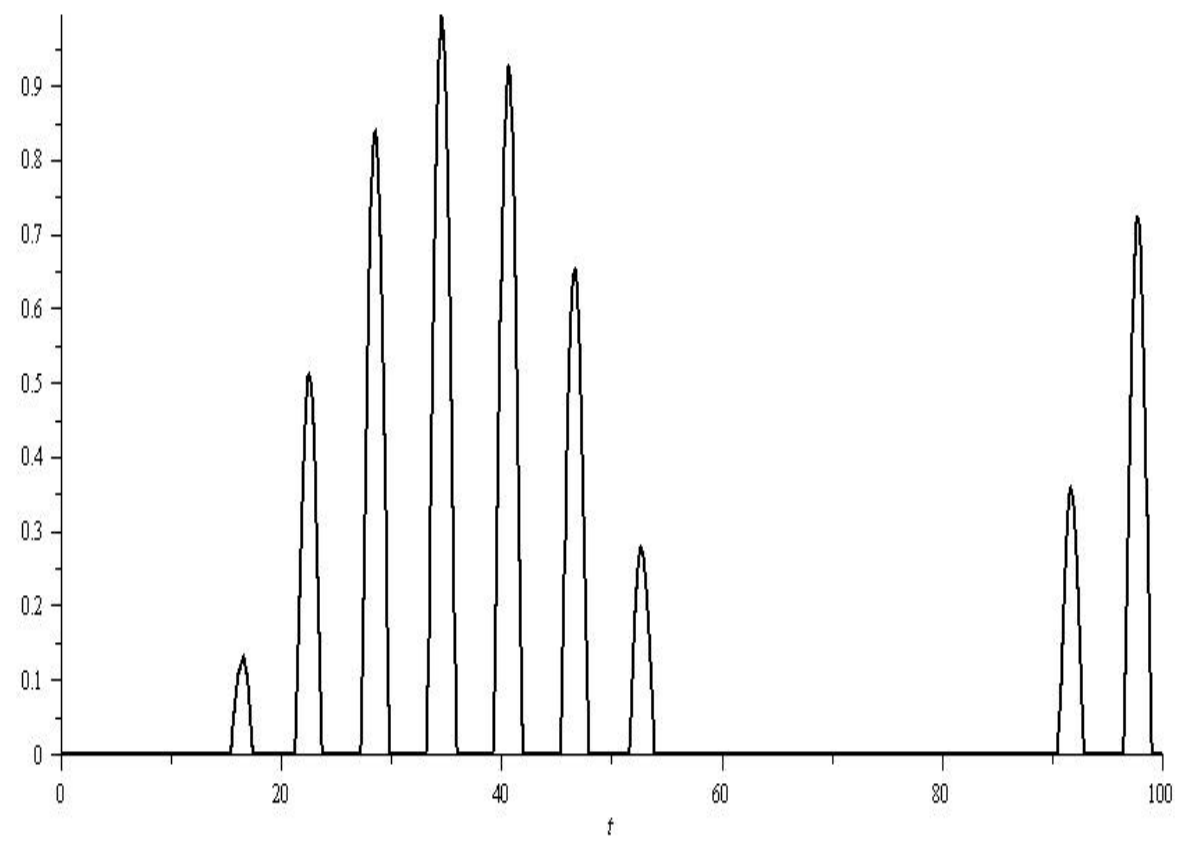

Figure 4. plot of $A_{2} B_{2}$ concurrence for $\alpha=\frac{\pi}{4}$ 


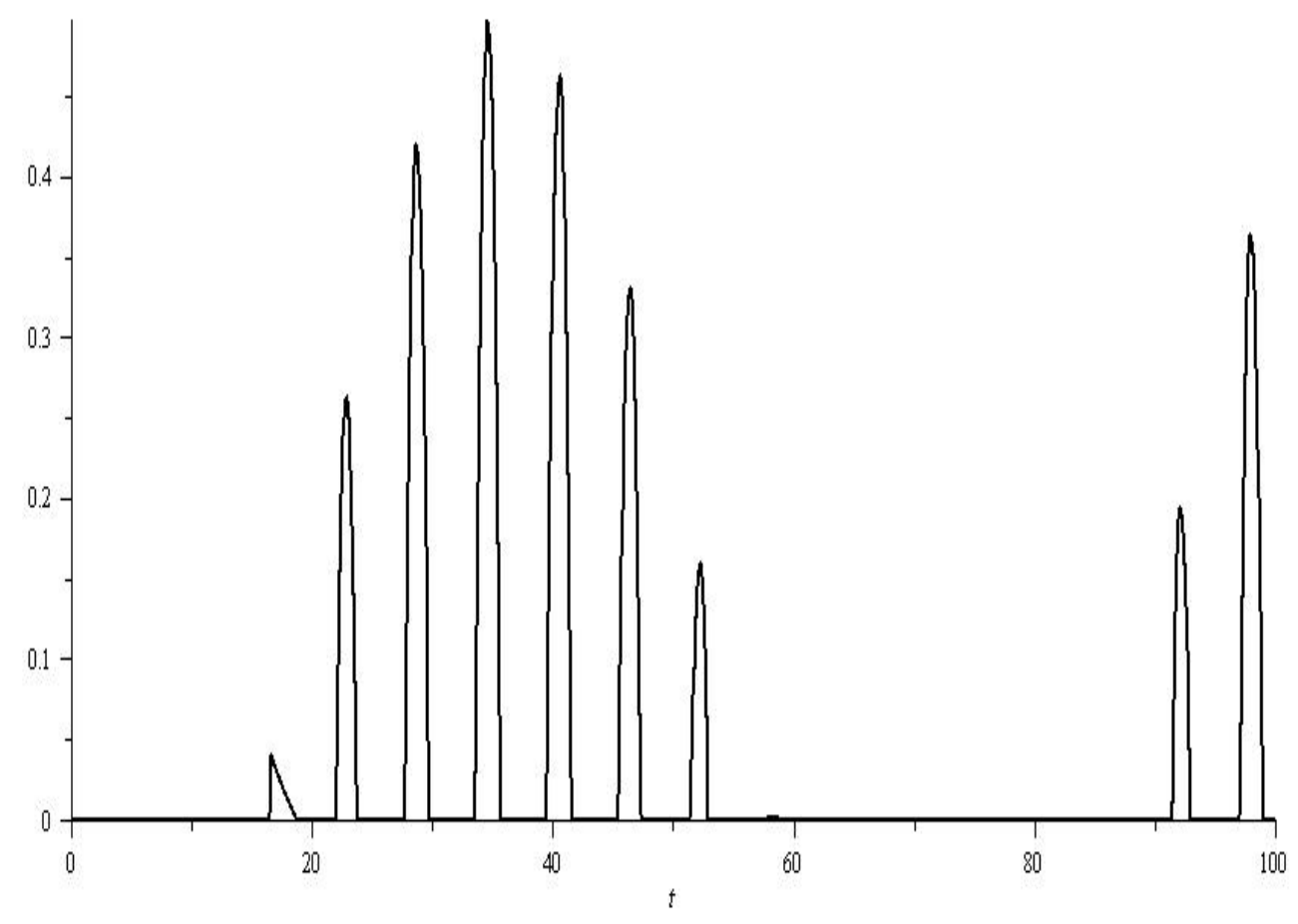

Figure 5. plot of $A_{2} B_{2}$ concurrence for $\alpha=\frac{\pi}{12}$

Now we move to concurrence calculation $C^{A_{1} B_{2}}$ and $C^{A_{2} B_{1}}$. We start with $C^{A_{1} B_{2}}$. For this, we trace out $A_{1}$ and $B_{2}$.

And we find

$$
\begin{aligned}
\rho^{A_{1} B_{2}}=\left\langle e_{2 A},\right. & \left.e_{1 B}\left|\rho^{\text {atoms }}\right| e_{2 A}, e_{1 B}\right\rangle+\left\langle e_{2 A}, g_{1 B}\left|\rho^{\text {atoms }}\right| e_{2 A}, g_{1 B}\right\rangle \\
& +\left\langle g_{2 A}, e_{1 B}\left|\rho^{\text {atoms }}\right| g_{2 A}, e_{1 B}\right\rangle+\left\langle g_{2 A}, g_{1 B}\left|\rho^{\text {atoms }}\right| g_{2 A}, g_{1 B}\right\rangle
\end{aligned}
$$

$$
\begin{gathered}
z=\left(x_{4} x_{5}^{*}+x_{6} x_{7}^{*}+x_{6} x_{8}^{*}+x_{10} x_{12}^{*}\right) \sin \alpha \cos \alpha \\
b=\left(\left|x_{2}\right|^{2}+\left|x_{3}\right|^{2}+|x 4|^{2}+\left|x_{6}\right|^{2}\right) \cos ^{2} \alpha+\left(\left|x_{7}\right|^{2}+\left|x_{14}\right|^{2}\right) \sin ^{2} \alpha \\
c=\left(\left|x_{2}\right|^{2}+\left|x_{3}\right|^{2}+\left|x_{4}\right|^{2}+\left|x_{6}\right|^{2}\right) \cos ^{2} \alpha+\left(\left|x_{8}\right|^{2}+\left|x_{9}\right|^{2}+\left|x_{13}\right|^{2}+\left|x_{15}\right|^{2}\right) \sin ^{2} \alpha
\end{gathered}
$$

Figures 6 and 7 show the plots for this concurrence for $\alpha=\frac{\pi}{4}$ and $\alpha=\frac{\pi}{12}$ at the first 100 seconds.

If we look at the in itial state in equation (19) we see that under the transformation $A \leftrightarrow B$ the state remain unchanged. That means $C^{A_{2} B_{1}}=C^{A_{1} B_{2}}$ at all times.

Similarly, we can find the explicit expressions for $C^{A_{1} A_{2}}$ and $C^{B_{1} B_{2}}$. The two cavities are not distinguishable, so we expect these C's to be the same and they are. The $\rho^{A_{1} A_{2}}$ has the form

$$
\rho^{A_{1} A_{2}}=\left(\begin{array}{cccc}
v & 0 & 0 & 0 \\
0 & w & z & 0 \\
0 & z^{*} & x & 0 \\
0 & 0 & 0 & y
\end{array}\right)
$$

The concurrence for this density matrix is[12]

$$
C=2 \max \{0,|z|-\sqrt{v y}\}
$$


Where

$$
\begin{gathered}
z=\left(\left|x_{2}\right|^{2}+2\left|x_{4}\right|^{2}+\left|x_{6}\right|^{2}\right) \cos ^{2} \alpha+\left(x_{7}^{*} x_{8}+x_{11}^{*} x_{12}+x_{12} x_{13}^{*}+x_{14}^{*} x_{15}\right) \sin ^{2} \alpha \\
v=\left(2\left|x_{6}\right|^{2}+\left|x_{10}\right|^{2}\right) \cos ^{2} \alpha+\left(\left|x_{3}\right|^{2}+\left|x_{14}\right|^{2}+\left|x_{15}\right|^{2}+\left|x_{16}\right|^{2}\right) \sin ^{2} \alpha \\
y=\left(\left|x_{1}\right|^{2}+2\left|x_{2}\right|^{2}+\left|x_{3}\right|^{2}\right) \cos ^{2} \alpha+\left(\left|x_{5}\right|^{2}+\left|x_{7}\right|^{2}+\left|x_{8}\right|^{2}+\left|x_{9}\right|^{2}\right) \sin ^{2} \alpha
\end{gathered}
$$

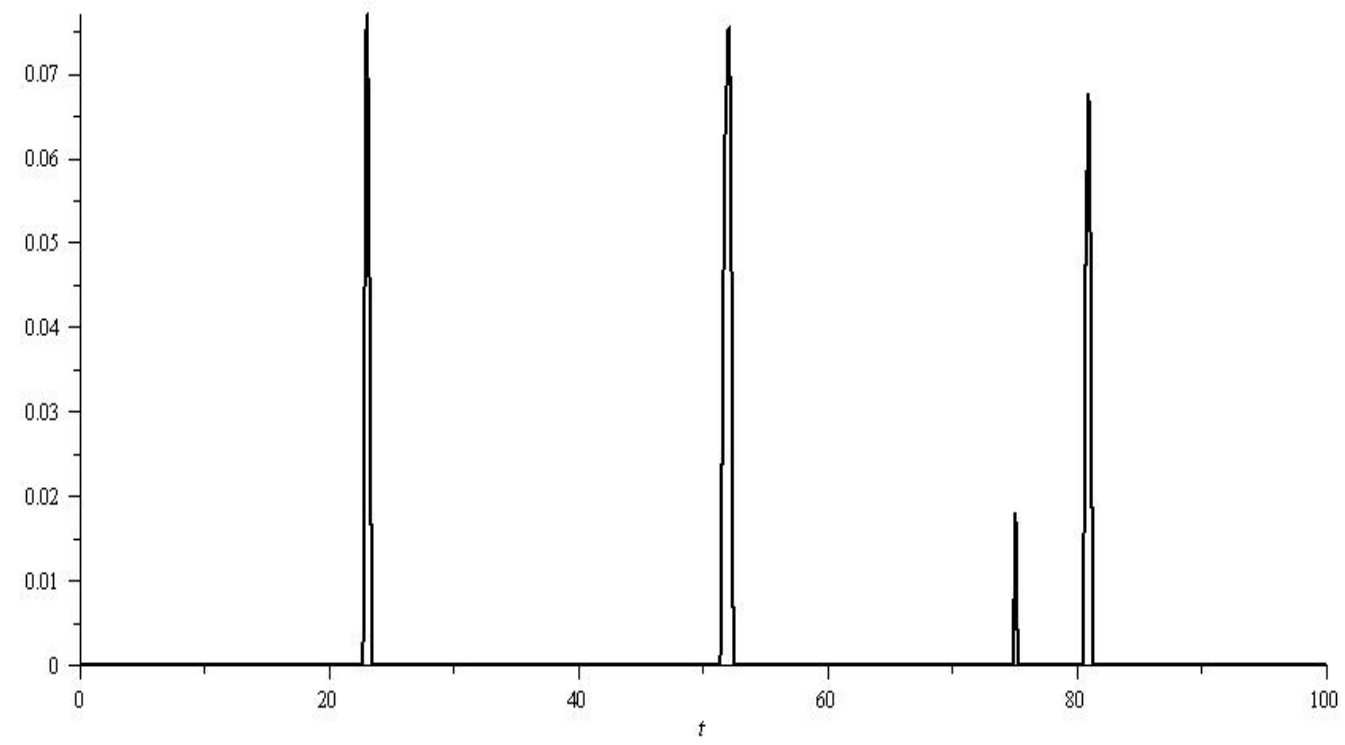

Figure 6. plot of $A_{1} B_{2}$ concurrence for $\alpha=\frac{\pi}{4}$

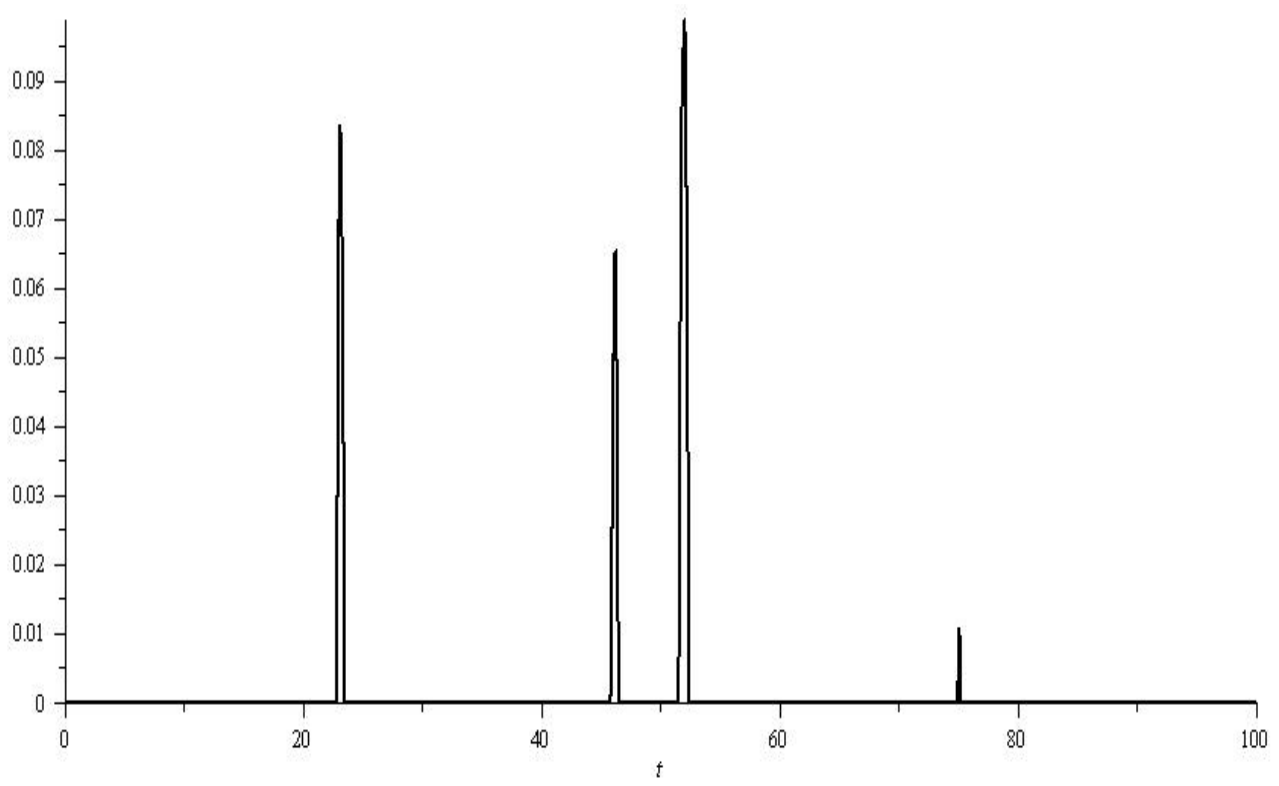

Figure 7. plot of $A_{1} B_{2}$ concurrence for $\alpha=\frac{\pi}{12}$

\section{Conclusions}

In this paper we considered two two-atoms Tavis-Cummings systems. These two systems have no interaction together, but one atom from each system are entangled initially ( $A_{1}, B_{1}$ ).
The entanglement between $A_{1}$ and $B_{1}$ for times $\mathrm{t}>0$ falls to zero and remains non-entangled for a period of time till becomes entangled again. We also see that the entangled intervals are smaller than non-entangled intervals. For initially non-entangled atoms, $A_{2}$ and $B_{2}$, the 
entanglement appears in short intervals. But the entanglement between $A_{1}$ and $B_{2}$ or $A_{2}$ and $B_{1}$ is very negligible in very short intervals; therefore, we can claim that these atoms will not be entangled afterwards.

We can test other initial states in our problem. For example for two initially entangled atoms, we can use another initially state

$$
\left|\Psi_{A_{1} B_{1}}\right\rangle=\cos \alpha\left|e_{1 A} g_{1 B}\right\rangle+\sin \left|g_{1 A} e_{1 B}\right\rangle
$$

and solve the problem again. Or for the non-entangled atoms, which we assumed that were in their excited states initially, we can change this prescription and solve the problem again, but the methods for this new situation are similar with the method which we used before.

\section{REFRENCES}

[1] McMahon, D.M., Quantum computing explained. 2008: Wiley-IEEE Computer Society Pr.

[2] Chuan-Jia, S., et al., Entanglement Dynamics and Bell Violations of Two Atoms in Tavis-Cummings Model with Phase Decoherence. Communications in Theoretical Physics, 2008. 49: p. 1505.

[3] Yönaç, M., T. Yu, and J. Eberly, Pairwise concurrence dynamics: a four-qubit model. Journal of Physics B: Atomic, Molecular and Optical Physics, 2007. 40: p. S45.

[4] Tavis, M. and F.W. Cummings, Exact solution for an N-molecule-radiation-field Hamiltonian. Physical Review, 1968. 170: p. 379-384.

[5] Tessier, T., et al., Entanglement sharing in the two-atom Tavis-Cummings model. Physical Review A, 2003. 68(6): p. 062316.

[6] Fujii, K., et al., Explicit Form of Solution of Two Atoms Tavis-Cummings Model. Arxiv preprint quant-ph/0403008, 2004.

[7] Zueco, D., et al., Qubit-oscillator dynamics in the dispersive regime: Analytical theory beyond the rotating-wave approximation. Physical Review A, 2009. 80(3): p. 033846.

[8] Keeling, J., Quantum corrections to the semiclassical collective dynamics in the Tavis-Cummings model. Physical Review A, 2009. 79(5): p. 053825.
[9] Vadeiko, I.P., et al., Algebraic approach to the Tavis-Cummings problem. Physical Review A, 2003. 67(5): p. 053808 .

[10] Amico, L. and K. Hikami, Integrable spin-boson interaction in the Tavis-Cummings model from a gen eric boundary twist. The European Physical Journal B-Condensed Matter and Complex Systems, 2005. 43(3):p. 387-391.

[11] Fujii, K., et al., Quantum Diagonalization Method in the Tavis-Cummings Model. Arxiv preprint quant-ph/0410003, 2004.

[12] Wootters, W.K., Entanglement of formation of an arbitrary state of two qubits. Phy sical Review Letters, 1998. 80(10): p. 2245-2248.

[13] O'Connor, K.M. and W.K. Wootters, Entangled rings. Physical Review A, 2001. 63(5): p. 052302.

[14] López, C., et al., Entanglement properties in the inhomogeneous Tavis-Cummings model. Arxiv preprint quant-ph/0611045, 2006.

[15] Zhang, G. and Z. Chen, The entanglement character between atoms in the non-degenerate two photons Tavis-Cummings model. Optics communications, 2007. 275(1): p. 274-277.

[16] Lian, J., Y. Zhang, and J.Q. Liang, Macroscopic Quantum States and Quantum Phase Transition in Dicke Models of Arbitrary Atom-Number. Arxiv preprint arXiv:1110.3862, 2011.

[17] Tsyplyatyev, O. and D. Loss. Dicke model: entanglement as a finite size effect. 2009: IOP Publishing.

[18] Latorre, J. and A. Riera, A short review on entanglement in quantum spin systems. Journal of Physics A: Mathematical and Theoretical, 2009. 42: p. 504002.

[19] Garraway, B.M., The Dicke model in quantum optics: Dicke model revisited. Philosophical Transactions of the Royal Society A: Mathematical, Physical and Engineering Sciences, 2011.369(1939): p. 1137-1155.

[20] Amico, L., et al., Entanglement in many-body systems. Reviews of Modern Physics, 2008. 80(2): p. 517.

[21] Bastidas, V.M., J.H. Reina, and T. Brandes, Non-equilibrium entanglement in a driven Dicke model. Arxiv preprint arXiv:0904.2411, 2009

[22] Reslen, J. and L. Quiroga, ENTANGLEMENT IN THE DICKE MODEL. REVISTA COLOMBIANA DE FÍSICA, 2004. 36(2). 